\title{
Structure of Relatively Accelerating Universe
}

\author{
Aloysius Sebastian \\ Via Martiri Oscuri 4, Milano, Italy \\ Email: aloysi.aloys@gmail.com
}

Received 17 January 2014; revised 15 February 2014; accepted 23 February 2014

Copyright @ 2014 by author and Scientific Research Publishing Inc. This work is licensed under the Creative Commons Attribution International License (CC BY). http://creativecommons.org/licenses/by/4.0/

\section{Abstract}

This article is about the structure of expanding universe. Here I am trying to create a structural model of universe according to my thoughts. Studies show that universe is accelerating its rate of expansion. Here I am trying to get some conclusions according to my thoughts. In my opinion, our universe is under a gravitational frame with all the matters in it. In this gravitational frame of universe we can consider our universe as a closed system and the empty space outside the frame as an open system. There are two possibilities we can find. One is relative motion of the objects inside the frame. By this way we can feel the universe is accelerating its expansion, but it is not. Another one is the vacuum energy absorption and negative pressure inside the frame. According to this thought, we can say that universe is accelerating and it can continue its motion at present conditions on it, even the force created by the big bang is decreasing. The other point is that, once the absorbed vacuum can create a negative pressure inside the frame, there is no need for the concept of dark energy in our universe [1]. According to this model, negative pressure inside the frame will be created by the absorbed vacuum from the space. So the existence of dark energy can be questioned. In this model, dark energy doesn't play any role in the expansion or rate of acceleration on the expansion of universe. So the dark energy doesn't exist in this model. During the absorption of vacuum to the frame, it will increase the volume of the frame. It will create free spaces inside the frame to accommodate the vacuum inside the frame. The rest of the absorbed vacuum will move to the center of the frame by the action against gravity and the force created by the big bang. It will increase the density of the absorbed vacuum in the center and can create the negative pressure on the frame to pull it outside to the empty space. It will cause the acceleration on expansion of universe. These things are explained deeply in this article. And here it also explains the destiny of universe according to the frame and expansion of universe.

\section{Keywords}

Structure of Universe; Relatively Accelerating Universe; Does Dark Energy Exist; Gravitational 


\section{Frame of Universe}

\section{Introduction}

We are having so many theories explaining the expansion and accelerating rate of the expansion of our universe. Here I am introducing a new model for the structure and dimensions of universe. According to this model I am considering our universe with a shape of a sphere and all the dimensions of a sphere. The universe before big bang is as a unique quantum closed system and the empty space is as an open system. After the big bang, the unique quantum (mass) with a unique gravity, will try to hold the split mass under a common gravitational field. In this model, universe is under a gravitational frame of all the matter present in it. Here I am trying to avoid the hypothetical concept of dark energy inside the frame of expanding universe. Vacuum can create a negative pressure against gravity, instead of dark energy. According to this, if vacuum can create a negative pressure, there is no need for a concept of dark energy in cosmology [2]-[14]. I intend to say that dark energy doesn't exist. According to this model the absorbed vacuum from the empty space will move to the center of the frame and fill inside the frame uniformly, by the increasing volume of the frame. The increasing volume of the frame will create more free spaces and it will allow the vacuum to stay inside the frame. We can imagine the future of universe with three types of ends.

\section{Expansion and Structure of Universe}

Here I am introducing a new model for the structure of universe [15]. To explain the structure of our universe here I would like to use the below mentioned figure (Figure 1, structure of universe).

As all we know that the origin of our universe is by the big bang. The explosion created by the big bang gives acceleration to the matter (energy) and all the matters present in the universe are started to move away each other. All we know universe is expanding. Also we know that the very huge mass which explore by the big bang has its gravity too. If we consider the huge mass as a unique quantum then the rest will be the empty space or vacuum energy. So we can say that the huge mass (unique quantum) was inside the empty space. So the unique quantum inside the empty space we can call as early universe before the big bang and I am not saying that it as the very first stage of universe. After the explosion the unique quantum was divided into several parts and starts to move away each other to the empty space (vacuum), by the force created by big bang. Once the divided parts of the unique quantum are moving to the space it is absorbing the empty space or vacuum to the inner side. Same time gravity of the total mass will try to attract each other.

Now we can consider the unique quantum (before big bang) which is staying in the empty space as a closed system. The empty space which allowing to stay the unique quantum as an open system. Now we can imagine

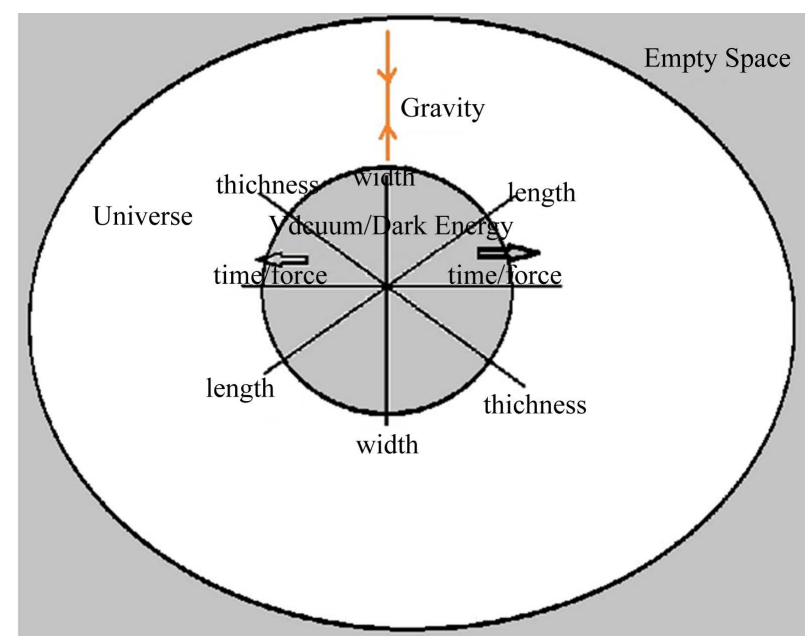

Figure 1. Structure of universe. 
the total system, at the center of the total system will be the unique quantum and rest will be the vacuum (empty space). The energy which concentrated at the center of the total system gets explored by the big bang, and moves away each other into the empty space (where the vacuum energy is present). After the big bang the closed system of unique quantum get changes. It will break and get motion into the open system by the empty space. But the gravity of the total mass present in the unique quantum tries to hold them together. So it will remain as a gravitationally closed system. According to me when the force created by the big bang gives a velocity to the matter (divided energy from the unique mass by the explosion created by the big bang) to expand into the empty space (vacuum energy), it will absorb the same amount of vacuum energy inside the gravitationally closed system. Now we can call it as a gravitationally closed frame of universe, where the matter (energy) presents. So if it moves to a distance of $1 \mathrm{~m}$ from the center of the frame it has to absorb the vacuum energy present in that distance to the center of the frame. So the absorbed vacuum energy will be move to the center of the frame and the moving matter (energy) will move to the empty space.

As I said before the total amount of the energy contains in the present universe was unique at past and had a common gravity to all these matter. After the explosion the gravity will try to unique them as like the past and acting against the force created by the big bang. But here we know the universe is still expanding and it accelerate its rate of expansion. Here we can see the energies are moving away each other to the infinite space, gravity is trying to hold them to the center and the vacuum energy is absorbing to inside of the frame. There is a gravitational force between all these energy or we can say the expansion of universe is under a gravitational frame. The action of gravity will cause the deceleration of the expansion of universe. But still the expansion of universe is accelerating.

Now we will start from the unique mass before the big bang. Once we are starting to create a structure we must be aware of its dimensions. We can consider the unique energy before the big bang as like a sphere with all its dimensions. Gravity will help it to obtain the shape. Now here we have the dimensions of our universe but now we have to know about the directions of matter after the big bang. After the explosion by the big bang, the force created will carry away the matter from the center. So the matter will move away from the center in all the dimensions of a sphere if it was in the shape of a sphere. Here time is an important object with all these dimensions. In the expanding universe, the time starts with the big bang and is present with the moving energy (matter). We can say that time is having its importance with all the dimensions. As I said before when it is moving away from the center the matter will absorb the vacuum to the frame according to its rate of expansion. So once it is moving away from the center and absorbing the vacuum to the center, the distance between the center to the moving energy will increase. That means the center of the frame will remain as empty and it can accommodate the absorbed vacuum from the empty space outside of the frame.

Here we are clear that the distances between the energies are also increasing during the motion, as what we are saying the universe is expanding. This I can explain with the help of a figure (Figure 2, Expansion of Universe).

The Figure 2 shows the expansion of two objects during the expansion. We are considering the unique matter before the big bang as in the shape of a sphere. Now we can project two objects from the surface of a sphere. Once these two objects travel the same distance from the sphere and reaches the points A and B accordingly at the same time, the distance between them will be d1. Again at the same interval of time these objects reaches a distance from the sphere $\mathrm{C}$ and $\mathrm{D}$ accordingly, the distance between them will be $\mathrm{d} 2$. Here we can see that $\mathrm{d} 2$ is greater than $\mathrm{d} 1$. In this situation, even if they are moving uniformly the relativity shows us the acceleration be-

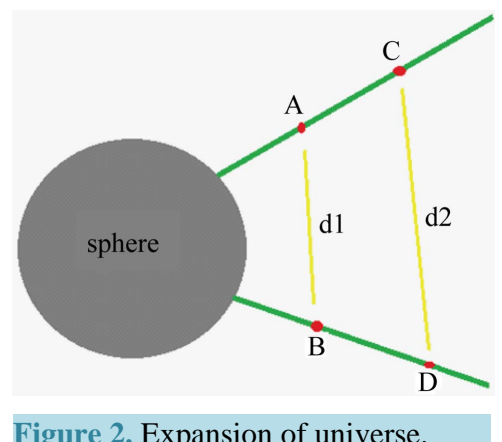

Figure 2. Expansion of universe. 
tween these two objects. Here we can clearly say that all the matter (energy) present in the universe is moving away from the center and also they are moving away each other.

Now we will discuss about the structural frame of matter (energy) in the expansion of our universe under the gravitationally closed frame. To explain this we have to consider gravity too. According to big bang, the total energy was unique quanta before the big bang. So the gravity was also unique. After the big bang, by the explosion created by the big bang energy is divided and gets accelerated. Naturally gravity also gets divided with the separated matter (energies). The separated energies get attracted during their acceleration too because there will be an attraction between two masses. This attractive gravity can create a frame of gravitation between all the energy present in the universe while they are moving also. So our universe is expanding with a gravitational frame. The important thing is that, the matter in the universe is still accelerating while gravity is acting on it. That I will explain below.

Now we will try to know about another possibility of the acceleration of the universe. We are clear that matter is getting accelerated in their directions. Where the matter (energy) is present will say as universe now. As I said before universe is under a gravitational frame. Once they are moving away to the empty space, they are absorbing the vacuum from empty space. We can call it as the coast of having space. The absorbed vacuum will remain in the frame and gets moved to the center of the total system, by the action of gravity between the matters present inside the frame. Here the force created by the big bang is pulling the matter away and gravity is holding them in the frame. So the region where the matter (energy) is present might that much hard and acts like a layer between the vacuum present in the center of the frame and the vacuum empty space outside the frame. But it can easily absorb vacuum from the outer empty space and passes it to the center like a medium.

Now we have to know about the areas of the frame and how it is helping to accelerate the expansion of universe. We can see that the area of the inner layer of the frame is less than always with the outer layer area of the frame. Here outer layer of the frame is absorbing vacuum from the empty space. The outer layer will absorb more vacuum, which the inner part cannot accommodate in. So once the absorbed vacuum will passes through the frame and accommodated to the center, the pressure inside the frame (from center to the inner layer of the frame) will be increase. This pressure can also pull the gravitational frame of universe away. According to this also the gravitational frame of the universe gets accelerated uniformly.

\section{Inertial Gravitational Frame of Universe}

Universe we can say as an inertial gravitational frame. All the objects in the universe are in a uniform gravitational field. So whatever particles (matter/energy) present in the universe are under the influence of a uniform gravitational field which created by the system. All the particles either microscopic or macroscopic are contributing their gravitational force to create a gravitationally closed universe. So all the particles in the universe are staying as gravitationally collaborated inside the universe. Another possibility for the expansion of universe we can say that, the Universe is uniformly moving with the gravitational frame. All the particles inside the Universe are at inertia (inertial motion) if there is no external force acting on it due to the gravitational frame. According to this way the total gravitational frame is uniformly expanding by the negative pressure from the center. During this expansion, the volume of the gravitational frame will increase. It allows more free spaces between the objects inside the gravitational frame of universe. These free spaces in the frame allow accommodating some amount of the absorbed vacuum from the empty space. As I wrote in the previous paragraphs, that the difference between the areas of the outer layer and inner layer of the gravitational frame. The outer layer area of the frame might much larger than that of the inner layer. So the outer layer of the frame will absorb more vacuum from the space, that the inner layer of the frame cannot emit it into the center of the total system. So the increasing volume of the gravitational frame will occupy the additional vacuum inside the frame. If the inner layer of the frame emit the same amount of vacuum which absorbs the outer layer of the frame, will leads the expansion of universe to a rapid acceleration rate. This will cause the destruction of the gravitational frame rapidly. Once the increasing volume of the gravitational frame allows accommodating some amount of vacuum inside the frame, it will control the rate of acceleration and the stability of universe. The increasing volume of the frame will create free spaces between the objects inside the frame and which allows accommodating the vacuum inside the frame; will affect the pressure of the frame. Here vacuum can create a pressure against gravity to help the expansion of objects inside the frame each other. So they can move away each other. In this form, we can observe that vacuum pressure is acting on the universe by two different situations. One is the vacuum pressure from the center 
of the frame and other is inside the frame. So here the external force for the expansion of universe is by the vacuum pressure. We can say this as a possible way for the acceleration rate on the expansion of universe.

Due to the Big bang a gravitationally Quantized single mass (unique quantum) was separated into several parts. By the force which produced by the Big bang, all these parts are moving away. This is our present concept about the expansion of Universe. If an external force acting on an inertial or non-inertial object, it will get accelerate by that external force. The force which acts on an object can pull or push the object. So the object will move with the force acting on it or the force is carrying the object with it. The force is with the mass (the distance from the starting point of the event and the time taken for travelling with the force is at same direction). The force, distance and the time are in the same direction. So the force which created by the Big bang is always with the motion of Universe (even with microscopic or macroscopic objects). The total mass of unique quantum has unique quantum gravity when the big bang occurs, and after the big bang the huge mass was split. But according to the long range of gravity, it still remains collaborated with the gravity of the total mass of the system. The gravity of the total mass is unified and creates the gravitational frame of universe. So the big bang can only break the system (it split the unique quantum), but the unified quantum gravity still remains as the same.

So the structure of Universe we can say like this way. If the universe is like football (with all the dimensions of a sphere) and which is having a leather layer with the thickness of 1 or $2 \mathrm{~cm}$. Now we can see all the parts of the football is under a frame. Inside of this 1 or $2 \mathrm{~cm}$ thickness will be the universe (gravitational frame). If we imagine our universe in the place of the football we can say that all the matter present in the universe is under a gravitationally collaborated frame. By this we will get a clear idea of the dimensions of universe (like an expanding sphere). If we consider our atmosphere as the empty space and the football without air as the gravitational frame of universe, and we are filling air to it. We can see that the air which it takes from the atmosphere will fill inside the frame and according to the pressure inside it starts expanding. It starts increasing its volume. We can consider our universe as like this, it is absorbing vacuum from the empty space and filling it to the center, by the increment of vacuum pressure it will expand to the empty space by increasing its volume. All the particles in the universe will be gravitationally collaborated and exist in this particular gravitational field which created by them together. Once it expands all the parts will expand in a uniform mode. So we can say all the galaxies stars even the smaller particle in our universe is in this region and are gravitationally attracted each other. Or we can say that universe is under a gravitational frame. Due to the force by the big bang when the universe is expanding and it expands together uniformly. This we can understand with the help of Figure 3, Gravitational frame of Universe.

Once it expands the gravity will keep the dimensions (like an expanding sphere) of the universe (gravitational frame) as the same and the volume of the particular region (gravitational frame) is increasing. The outer part of this gravitationally attracted universe expands to the vacuum and absorbs the vacuum energy and emits it to after the inner part of the universe (the region after the gravitationally attracted frame of universe or center of the frame). The outer layer absorbs more vacuum. The inner layer cannot emit (to the center of the frame) the same amount of vacuum which absorbs the outer layer. According to the increment of volume of the gravitational frame, there will be more free spaces which can accommodate some amount of the absorbed vacuum. The rest will emitted to the center and the pressure on the center will increase. So it will pull the frame for an expansion. The universe is expanding because the volume of the universe is increasing. So the present universe is staying in between these two vacuum quanta. So when the volume of the Universe (the region where the gravitationally quantized energy presents) increases the objects inside the frame can move away each other, because the increasing volume will create free spaces between them. These free spaces will be filled by the vacuum and act against gravity too.

The other side distance from the center to the inner layer of the frame is seemed to be increasing. If we are thinking about the dimensions on the expansion of universe, we can say our universe is expanding like an expanding sphere. It is having all the dimensions of a sphere. Here I am trying to explain it with the help of a Figure 4, Dimensions of Universe.

In the Figure 4 the dimensions like length, width or thickness all these are straight with their direction. Here the time and fore is always accompanied with these dimensions. The initial force for their motion and the time starts with the big bang. These all are moving away each other and the gravity on the opposite direction to all these. The big bang breaks the initial unique quantum gravity by the unique quantum and the gravitational force of the total system is trying to achieve its past sate. We can say that the early universe before the big bang as a unique quantum, and gravity is trying to reform universe as a unique quantum again. So here we can say that 

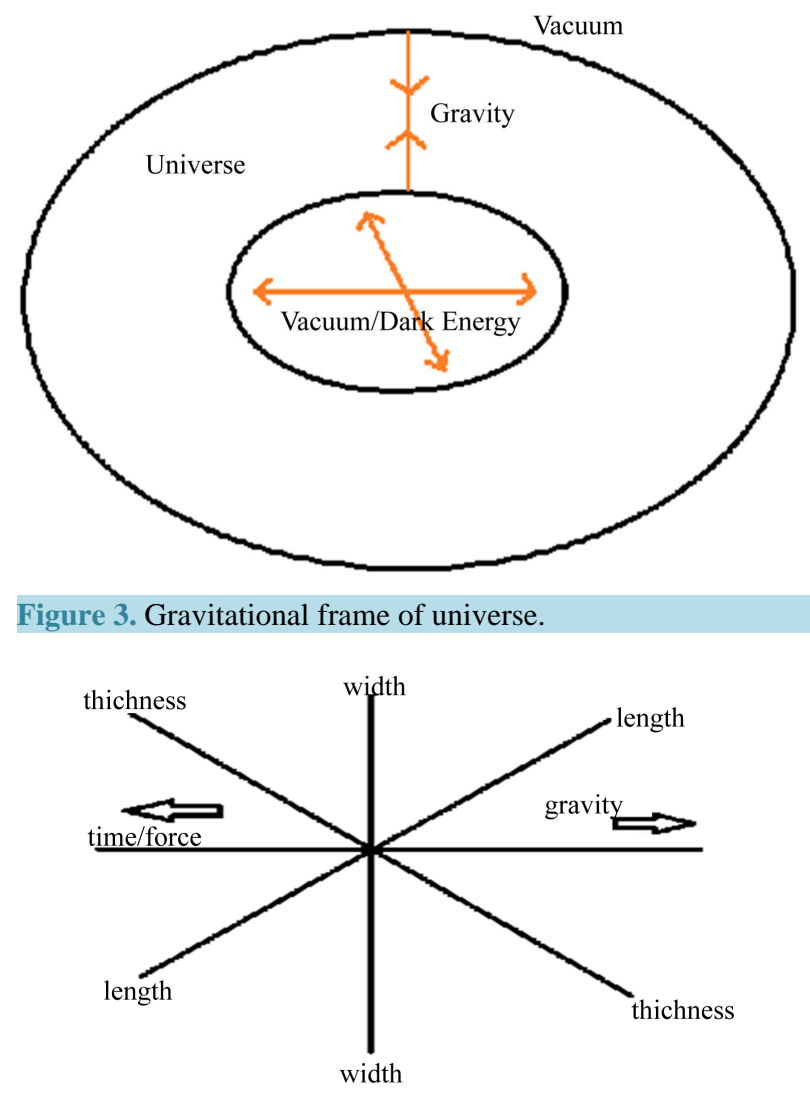

Figure 4. Dimensions of universe.

gravity is against to all the dimensions of the expanding universe.

\section{Relativity and Expansion of Universe}

Here I am trying to explain about the acceleration of the expanding universe. I am not going to start a complicated discussion about the acceleration rate of the expansion of universe. I would like to say that if the expansion of universe is at a constant speed, also we can feel it as accelerating because of relativity. It is just a possibility and I am not arguing it as the one correct to explain the acceleration rate of expanding universe. Sometimes relativistic motions of the objects can show us that they are accelerating. Here I am trying to explain it according to my point of view. Everything in the universe (even us) is moving according to the expansion of universe. I will explain it with the help of a Figure 5, relatively accelerating Universe. The Figure 5 shows us the relativistic motion of three objects.

As all we know universe is expanding and continuing its expansion with an acceleration rate. Here I place this figure to get a clear view. According to the Figure 5 there are three objects starts moving with a constant velocity. Here three objects "A", "B" and "C" start moving from a point "S". The objects "A" and "C" start moving with the constant velocity from the point $\mathrm{S}$ and the object " $\mathrm{B}$ " will start after a time interval. We can see that the object " $\mathrm{B}$ " is travelling in a straight line objects " $\mathrm{A}$ " and " $\mathrm{C}$ " are also travelling in straight lines and keeps same angle related to object "B". After a time interval " $T$ " these objects " $\mathrm{A}$ " and "C" will be reaching at a distance " $\mathrm{D}$ ". The distance between "B" to "A1" and "B" to "C1" are equal. It is clear that they all travel at same distances because the velocity is equal. This point we can say they reach at "A1" and "C1". Here we can find a distance between "A1" and "C1". Once they reaches at the point "A1" and "C1", the object "B" starts moving at the same velocity which have the objects "A1" and "C1". Here we can say these three objects are moving at same velocity and will travel same distances during a time interval.

The roll of relativity will starts now and we can feel the objects " $C$ " and " $\mathrm{A}$ " are accelerating related to the object "B" even if they are travelling at same velocity and same distances at same time. The object "B" also will 


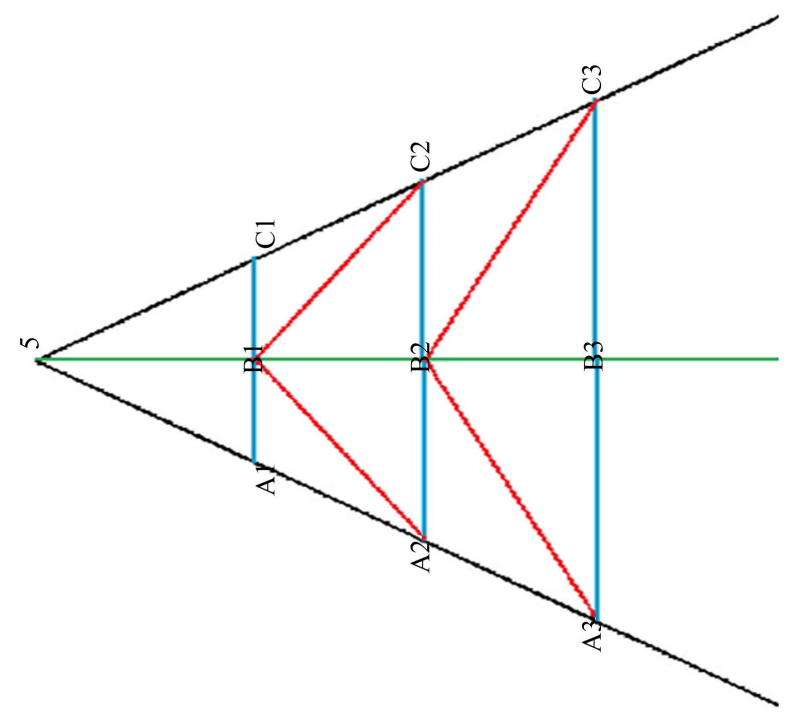

Figure 5. Relatively accelerating universe.

take the same time to reach the point "B1", which was taken the objects " $\mathrm{A}$ " and "C" to reach the points "A1" and "C1". Once the object "B" reaches to the point "B1" the other two objects "A" and "C" will reach at "A2" and "C2". The time taken by these three objects is equal. Now the object "B" is at the point "B1", "A" at "A2" and "C" at "C2" respectively. Here comes the roll of relativity. Here we can find three types of distances even they are travelling at same velocity. The first one is the total distance travelled by each object. The second one is the distance between "A2" and "C2". The third and important one is the distance between "B1" to "A2" and "B1"to "C2". Here we can find that the distance between "B" to "A1" is lesser than with the distance between "B1" to "A2", even they travel at constant velocity and same distance. So according to the distance travelled by each object, the distance between them will increase.

In this stage we can see the distance between the objects varies and increasing. The distance between " $\mathrm{A2}$ " to "C2" is greater than "A1" to"C1". At the same time the distance between "B1" to "A2" and "B1" to "C2" will increase. This is the important think at this motion, because of the increment of the distance between the objects even they are travelling at constant velocity. These things we can calculate with the help of trigonometric equations easily. The Figure 5 which I placed here will allow more clarifications.

Now we will come to our topic. Here we can consider earth as the object "B" and two distant galaxies or stars as object "A" and "C". So an observer from earth can feel that these two distant stars are accelerating its motion even if they are moving at same velocity. So here we may be able to say that the universe is not accelerating its motion. We feel it due to the relativistic motion of the objects in the universe. It is just a possibility only. Up to now we are unable to find the secrets of our universe.

\section{Dark Energy (Vacuum Energy Expansion)}

In the inertial gravitational frame, the outer layer of the frame absorbs the vacuum energy from the empty space. The inner layer emits the absorbed vacuum energy to the center of the gravitational frame (inner side after the frame), because gravity of the frame will pull it into the center of the frame. The area of the outer side of the frame is greater than that of the inner side. So the outer side of the frame will absorb more vacuum energy to the frame and the inner side of the frame must have to accommodate those absorbed energy to the center of the structure. This will cause the increment of density of vacuum energy in the center (after the inner side) of the frame. Increase of density of vacuum energy will create an increment of pressure at the center of the frame. This negative pressure created by the vacuum energy will pull the inner side of the frame against the gravity. It will cause the expansion of the volume of the frame. The gravitationally collaborated frame of the universe can only expand to the empty space. During expansion it absorbs vacuum energy again and it continues as a regular process. It is the reason of expansion and acceleration process of our universe. This is what I am trying to explain below. 
As I said before our universe is gravitationally collaborated with all the forms of matter or energy exist in it. There is a huge amount of vacuum energy (absorbed from the empty space) exist inside the frame of present universe. After the frame is the empty space which the gravitational frame of universe expands. Normally peoples say this vacuum energy as negative energy which create negative pressure against gravity and the other forms or energy in the universe as matter (energy). Our universe the amount of energy is finite and conserved which can be neither created nor destroyed. The presently accepted theory of origin of universe is the big bang theory. Here I also would like to work with the big bang theory. As all we knows the universe was created by the big bang happens in a unique quantum, and it starts expanding. According to me, during the big bang or before the big bang the position of the unique quantum was somewhere in the huge empty space (vacuum). So here we can say that, the total energy in the universe will be present inside a huge vacuum empty space.

In the Figure 6 (Initial Universe), the black part is the huge amount of vacuum empty space and the white part is the total energy (unique quantum before the big bang).

Once the big bang occurs the energy (unique quantum) starts expanding into the empty space or vacuum. As I said before once an object gets motion when a force acting on that object, the force will carry that object or the force is moving with the object. Here the force is created by the big bang to the unique quanta (the total energy or matter present in the universe). We can say that gravity is the only force hold the total energy (unique quantum) before the big bang. Once big bang occurs the unique mass (unique energy) or the total energy get explored and gravity of the total mass tries to hold them unique. But the force created by the big bang tends the separated matter (energy) to expand to the huge empty space (vacuum). Here we can assume that the unique quantum was in the shape of a sphere because of the attractive force of gravity. So the explored moving quanta of all the energies will get all the dimensions of a sphere. Now we can say that, our expanding universe is having all the dimensions of a sphere or it expands like a sphere expands.

According to me gravity will try to hold the objects in a system when they are separate by any another external force act on it. Once we heats water it will boil and then vapored. If we observe the water vapor we can see that the vapor is not separating away. It is because of the gravity of the water molecules in the vapor (system) tries to hold them together. Here the thermal force is acting on the water to separate the molecules but the gravity tries to hold them.

So here I can say that the force created by the big bang tends the total matter (energy) to separate and the gravity tries to keep them together. But here the matter (energy) can expand to the vacuum (empty space). So if it expands the system needs to absorb vacuum energy to it, according to the rate of expansion. The system has to accommodate the absorbed vacuum energy in it. According to these actions between the force by the big bang and the gravity of the system will allow creating the structure for the system, to accommodate the absorbed vacuum in it. The force by the big bang allows the system to expand (absorb) to the vacuum (empty space) and the gravity will pull the absorbed vacuum to the center of the system because gravity needs to hold the matter or energy inside the frame. Once the vacuum moves to the center, it will increase the vacuum pressure over there. It will create a negative pressure against gravity inside the system. These two actions will help the total system to form a structural frame and the universe gets its structure itself by these two actions. That I will explain with the Figure 7, Universe on Expansion.

In the Figure 7, the black portion in the outside is the empty space or vacuum energy. White portion is the region where the energy presents with the attractive force of gravity or we can call as observable universe. The black portion inside the frame is the region where the absorbed vacuum energy will be accommodated. Consider

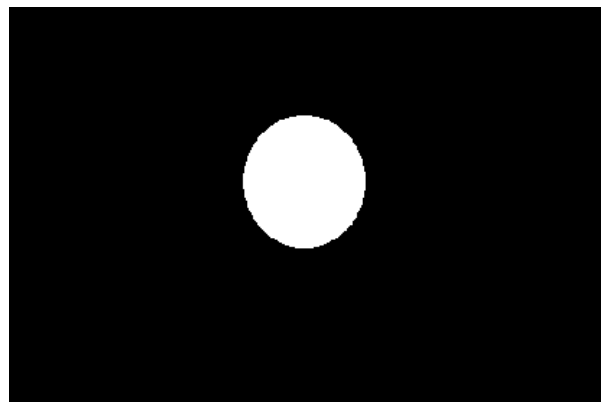

Figure 6. Initial universe. 


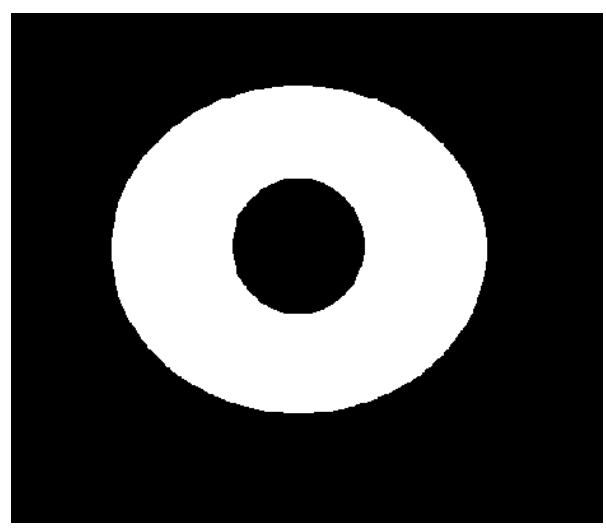

Figure 7. Universe on expansion.

this figure as a sphere expanding to the empty space and imagine that inside this sphere there is another sphere is creating by the absorbed vacuum from the empty space. In between these two vacuums there is a sphere of quanta (matter or energy) and with all the dimensions of a sphere.

In this figure we can see that the region where the matter is present has two ends. One leads to the biggest empty space and the huge amount of vacuum energy. So once the universe is expanding, the outer part of the frame will absorb the negative vacuum quanta from the empty space to inside of the frame. The inner end of the frame (white portion) in the Figure 7 touches with the region where the absorbed vacuum energy is accommodated. This end will emit the absorbed vacuum energy to the center of the total system where the vacuum energy will be accommodated. As I explain before inner shell of the frame cannot emit the whole vacuum to the center at the same time. It will hold some amount of absorbed vacuum inside the frame. This process will continue.

In this figure we can see that the "area" of the end of the frame which absorbs vacuum from the empty space is greater than that of the end which emits the vacuum energy to the center. So that the outer parts will absorbs more vacuum energy to the frame (observable universe) and the gravity will pull it to the center of the frame because gravity needs to hold the total quanta of energy present inside the frame to its potential attractive fields. Once at the center is filling with the vacuum the density of the center where the vacuum energy is filled will increase. The increased density will create a negative pressure against the gravity to pull the gravitationally collaborated matter (scattered Quanta by the big bang) region to expand. By this it will increase the volume of the frame will increase. It will increase the expansion rate of the universe faster than previous. We can say at this stage cosmic acceleration starts. That means that, when the gravity starts to pull the vacuum to the center of the frame and when the vacuum pressure increase and starts to pull the frame upwards, starts the cosmic acceleration. It is continuing as a regular process now onward. So I can say that at present, the expansion of universe can go on without the force created by the big bang. I am not saying that the force created by the big bang is not helping the expansion of universe, but I can say that universe can expand itself by the absorption of the vacuum from the empty space and the negative pressure against gravity of the total mass present in the observable universe. And this is the answer, if someone asks why cosmic acceleration begins.

\section{Does Dark Energy Exist?}

As all we know that dark energy is a hypothetical concept of energy in cosmology, which is filled in the space uniformly and tends to accelerate the expansion of universe by acting against gravity. It is creating a negative pressure against gravity and encourages the expansion rate of universe. There are so many theories explaining dark energy. I am agreeing with these theories which they are trying to prove the properties of dark energy, but I am not agreeing with the hypothetical model of dark energy. As I wrote above, if the absorbed vacuum from the empty space can create a negative pressure against gravity and encourage the acceleration on the expansion of universe, there is no need of a hypothetical model of dark energy. I would like to say that, dark energy does not exist. Vacuum energy can create a negative pressure against the gravity to accelerate the expansion rate of universe. According to cosmological constant, dark energy is uniformly filling in the space. As I wrote above we can see that the vacuum is uniformly filling in the universe and the effect of gravitational force it is moving back to the center of the frame. When observable universe expands it volume it will create some free space and the 
vacuum will fill in it uniformly. According to me the absorption of vacuum from the empty space, filling it into the universe and moving back to the center of the frame by the influence of gravity, are a regular process in our universe. Here we can see the conclusions of cosmological constant are right, if it mention about the absorbed vacuum from the empty space. The quintessence model shows us the density of dark energy varies according to space and time. Even it is uniformly filling in the universe; the density of vacuum content can varies according to the gravitational influence in the space. The strong gravitational fields can act more against the absorbed vacuum and it will cause the density difference of vacuum in strong gravitational fields and weak gravitational fields. Our universe is expanding according to the time started with the big bang, so the occasions and places will vary according to time. We can say clearly that, these theories are right if they explain the properties of absorbed vacuum quanta from the empty space. Or otherwise we have to say that the expanding universe is absorbing some kind of energy instead of vacuum and this is what we can call as dark energy.

We can consider our universe under the gravitational fame as a closed system, which expands to the empty space (to an open system). Once it expands, naturally its volume also gets changes. It is expanding to the empty space and absorbing vacuum from the empty space to the gravitationally closed universe. Here the cost of having space is equal to the absorbed amount of vacuum. That means, the amount of quanta of energy expand to the empty space is equal to the amount of vacuum or empty space absorbed. So the absorbed vacuum can create an equal negative pressure inside the frame of universe. The rate of empty space occupied will be equal to the rate of quantum vacuum occupy inside the frame of universe. This will keep the orders of magnitude as zero. The cosmologic constant remains equal to the large term of the opposite sign.

\section{Destiny of Expanding Universe}

In this part I would like to discuss about the destiny of our expanding universe. We all are confused about this subject. We are having so many suggestions and explanations about this subject by different scientists. All we know the universe is expanding like an expanding balloon with all the four dimensions. Here I am going to discuss about the concept on the structure of universe. I was explained a structural model and dimensions of universe before. According to me our universe is under a particular structural frame and the matter is present at this frame only with the strong influence of gravity. The expansion of universe will leads to the absorption of empty space (vacuum energy) into the frame. The center of the frame is filled with vacuum energy and the increasing density of the vacuum energy in this region will increase the pressure here and cause to increasing the acceleration rate on expansion of the universe. But in the other case, if the universe is expanding like an expanding balloon, explaining us entire volume of universe can contain the matter and the dark energy is filling in it uniformly. In an open universe like this cannot create a negative pressure against the gravity so far. According to me the matter will be present in the frame but the volume of the universe may equal in these two models.

I am also agreeing with the big bang theory. The big bang produces a force which carries the matter away from the center where the big bang occurred. According to me the force must carry the matter from the center and the center will be empty due to this case. Once the matter move away from the center to the empty space, the absorbed empty space must be move to the center. So here I am saying that the center of the universe must be filled with empty space (vacuum). If the universe is expanding like an expanding balloon the center will never remain as empty with high pressure. The vacuum will be filling uniformly in all the regions and the pressure never increase uniformly because the universe is open and expanding. Here the pressure remains constant at all parts so the pressure can act on all dimensions. At this situation the pressure can pull the matter to the empty space outer dimensions, but at the same time there we can observe another possibility too. If the vacuum energy is filling in this way, the pressure can push the matter to all the dimensions of the universe, even to the center. It will reduce the expansion rate of universe.

The Figure 8 (Negative pressure), we can imagine the dark area as the vacuum energy and the white circles as the galaxies or stars. The arrows will show the directions which can pull the pressure of the vacuum energy.

According to the structure of the universe which I explained before will not allow this problem because the vacuum energy will be move to the center of the frame of the gravitationally closed universe. So the pressure occurring by the increment of vacuum energy inside the frame will only pull the frame on to the outside empty space and absorb more vacuum energy into the frame. Due to this pull the frame will increase its volume. This increment of volume of the frame will cause the expansion of universe or the increment of the distance between two distant galaxies which we can observe. 


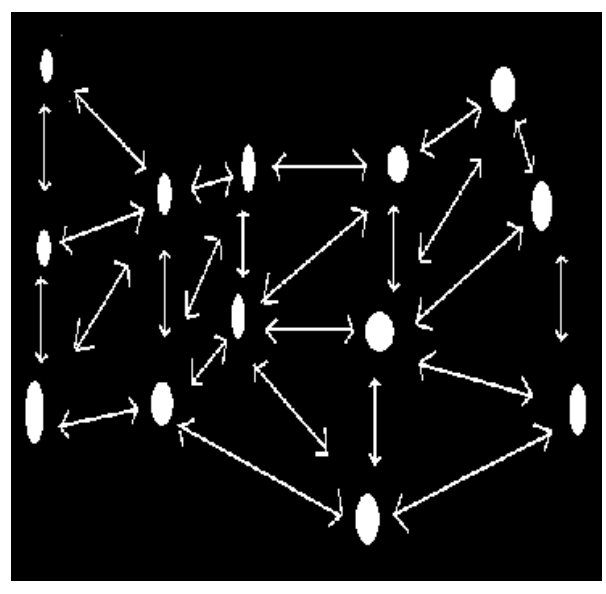

Figure 8. Negative pressure.

Now I would like to discuss about the destiny of the universe. According to me there will be three possibilities on the destiny of the universe. They are the static universe, isolated universe, and big crunch of all the matter present in the universe accordingly. We all are familiar about these three possibilities, but here I am explaining these three possibilities according to the structure which I explain before.

\subsection{Static Universe}

Static universe is a possible proposal for the destiny of the universe, if it stops its expansion to the empty space. Once it stops its expansion it will never absorb vacuum energy more. So the vacuum density and negative pressure in the universe will remain as constant. If the negative pressure is not pulling the matter, it can remain where it is. To explain this we have to look at the frame of the universe which I explain before. Due to the pull of the negative pressure the frame have to expand and the volume of the frame must be increase during the expansion. This expansion may cause to grand decrement of the dimensions of the frame.

In the Figure 9 (Static Universe), the dark area is the empty space and the first part of the figure we say as the universe at present. The second part we can imagine as the static universe which created by the end of the expansion of universe. The static universe may be created by the effect of gravity and the vacuum energy (negative) pressure. As all we know that the expansion of universe is increasing the volume of the universe. During the expansion, distance between the objects (e.g. Galaxies, stars ......) is increasing. So the gravitational force between them also undergoes weak. After a particular stage it will affect the stability of the gravitational frame of the universe. At this time gravity may play a crucial role on the frame to keep the energy in the frame. Gravity will try to reduce the dimensions of the frame and became thinner. Once gravity is trying to reduce the dimensions of universe, it will affect the vacuum energy pressure inside the frame. It reduces its pressure inside the frame. This will cause the accelerating rate of the expansion of the frame. The rate of acceleration will be reducing at this stage. After continuing this process the frame will became thinner and the acceleration of the frame will be stopped. Gradually it will stop the expansion also. Once the frame is not expanding the universe will became static. The frame will stop its expansion and all the matter in the frame will stay at the place where it is at the stage when it stops expansion. The vacuum energy density and pressure rest as constant. After that the percentage of everything like matter dark matter or vacuum energy are remain as unchangeable.

\subsection{Isolated Universe}

We can think about an isolated universe model also at the destiny of our universe. It is happens when the gravitational force of the frame breaks up. As I said before once the universe expanding its volume, the gravity between the mass (energy) is going weaker and weaker. After a certain volume the gravitational force can't hold the matter inside the frame because the frame will expand more than the range or gravitational fields. At this time the gravitational frame may be broke and the distance between two distant galaxies may be more than that of their gravitational force can attract each other. So at this stage these two galaxies are free from each other they do not show any relations with the help of gravity. This stage we can say that these galaxies are isolated 


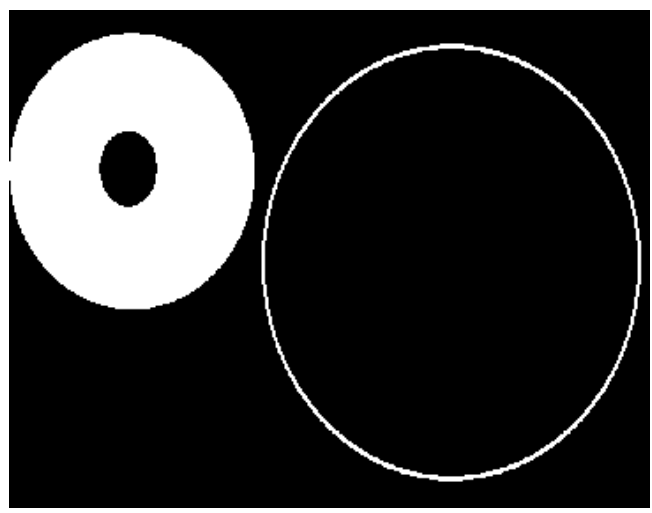

Figure 9. Static universe.

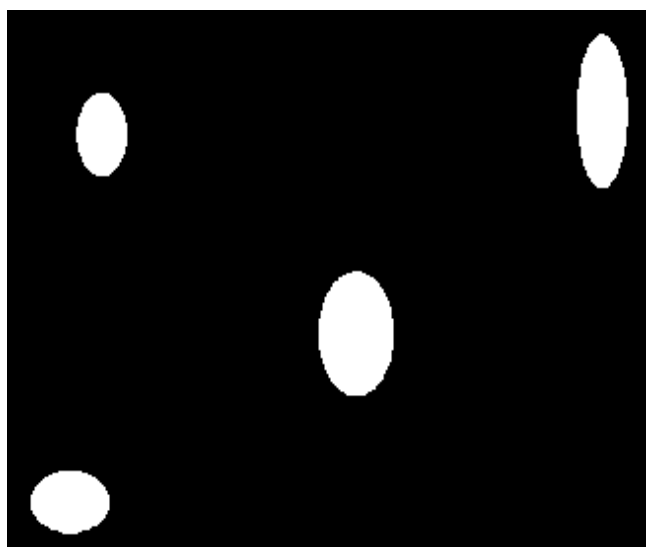

Figure 10. Isolated universe.

each other.

It may happen in the universe future because our universe is still expanding. So each isolated universe we can call as a single static universe. The total gravity of each isolated static universes are concentrated in the center of the galaxy. The gravitational break up may can create a force to pull each isolated universe each other. This stage we may can say another big bang is occurring in the universe. But it is happening in the frame of universe. It may happen because the gravitational break up will break the frame too. So if the frame broke the pressure inside the frame will release very fast. These two things will happen at the same time and it will produce a more powerful force on the matter (energy) in the gravitational frame of the universe. This we can say as the big bang by the pressure explosion because the sudden variation of pressure is creating this big bang.

By the Figure 10 (Isolated Universe), the dark area is the empty space and the white circles are the isolated universes. In between them there are no gravitational attractions.

\subsection{A Big Crunch of All the Matter Present in the Universe}

A big crunch of all the energy present in the universe is also a possible way to the future of our universe. According to me it is the most possible way for the future of our universe. Here also I want to say what I said before, the expanding volume of the universe will reduce the attractive force of gravity between two distant galaxies. This will cause the gravitational break up. Once gravitational break up happen the vacuum energy inside the frame can easily exit from the frame. As a result of this the pressure inside the frame of universe will decrease. The flow of vacuum energy from the frame to out of the frame will pull the energy to the center and gravity of the total mass will help this process. According to these two actions the motion of matter to the center will accelerate. Finally all the matter will collide together at the center to form as a unique quantum. But the high velocity of moving matter to the center may cause another big bang. So the universe may undergo a big crunch and then a big bang. 


\section{Acknowledgements}

I wish to thank Ms. Hiromi Takashima (Hakodate, Japan) for supporting and funding me during this period. I wish to Thank Mr. Alline Xiao for his great advice to me to systemize this paper perfect.

\section{References}

[1] http://en.wikipedia.org/wiki/Dark_energy, articles in Wikipedia English and Italian.

[2] http://en.wikipedia.org/wiki/Physical_cosmology, articles in Wikipedia English and Italian.

[3] http://en.wikipedia.org/wiki/Hubble\%27s_law, articles in Wikipedia English and Italian.

[4] http://en.wikipedia.org/wiki/Universe, articles in Wikipedia English and Italian.

[5] http://en.wikipedia.org/wiki/Ultimate fate of the universe, articles in Wikipedia English and Italian.

[6] http://en.wikipedia.org/wiki/Big_Bang, articles in Wikipedia English and Italian.

[7] http://en.wikipedia.org/wiki/General relativity, articles in Wikipedia English and Italian.

[8] http://en.wikipedia.org/wiki/Plank\%27s_constant, articles in Wikipedia English and Italian.

[9] http://en.wikipedia.org/wiki/Newton\%27s_law_of_universal_gravitation, articles in Wikipedia English and Italian.

[10] http://en.wikipedia.org/wiki/Quantum_field_theory, articles in Wikipedia English and Italian.

[11] http://en.wikipedia.org/wiki/Quantum_mechanics, articles in Wikipedia English and Italian.

[12] http://en.wikipedia.org/wiki/Uncertainty_principle, articles in Wikipedia English and Italian.

[13] http://en.wikipedia.org/wiki/Space-time, articles in Wikipedia English and Italian.

[14] http://en.wikipedia.org/wiki/Lorentz_transformation, articles in Wikipedia English and Italian.

[15] http://en.wikipedia.org/wiki/Standard_model, articles in Wikipedia English and Italian. 